\title{
Rouh/Qi before and after production; basic concept of life
}

\begin{abstract}
Any cells in the world has life at the level of microsystem or macro system, which based on concept of traditional medicine like Persian Medicine (PM) and Traditional Chinese Medicine (TCM) formed by 4 elements: Wetness, Coldness, Warmness and Dryness in PM and Blood, Yin, Yang and Qi in TCM. Any interactions among these four elements lead to production of Rouh (PM) and Qi (TCM). Rouh/Qi is source of viability of cell, human, animal, plants and so on. In our previous basic studies the overall meaning of Rouh/Qi and other elements was described clearly. In this report there is an effort to focus more than before on mechanism and outcome of Rouh/Qi production.
\end{abstract}

Keywords: persian medicine, chinese medicine, production, meraque, rouh, qi, mezaj, picture of life, zheng
Research Article

Volume II Issue 2 - 2018

\begin{abstract}
Avijgan Majid, Avijgan Matab
Department of Infectious Disease and Persian Medicine, Isfahan University of Medical Sciences, Iran
\end{abstract}

Correspondence: Avijgan Majid, Department of Infectious Disease and Persian Medicine, Isfahan University of Medical Sciences, Iran, Tel +4676 I I 96 285, Email avijgan@yahoo.com

Received: November 16,2017| Published: March 06, 2018

\section{Massage}

Rouh/Qi production is the first step of energy generation which by distribution and reaching to cells of human body will provides life and or living.

\section{Introduction}

This is a hypothetical review based on library studies. What is life and viability? What agent is necessary for viability? This is a major question which is not yet clear in biomedicine and western medicine. But this item has clearly described in more than 3000 to 4000 years ago (In Traditional Medicine) when there was not any laboratory. This is an important issue that the world and cosmos is depend on Rouh/Qi which needs to have a clear description. In religious believes, there is a kind of Rouh which has been given by God to human. It is spiritual Rouh (Original Qi, Ethereal). This is accepted by all religious schools. But when non-religious peoples ask about the viability, they need to have an answer for accepting vital process. In response to them; there is another Rouh (Qi) which originated from spiritual Rouh/Qi (depend to it) but has specific character which both of them have main role in life and living. ${ }^{1-4}$ The production of this Rouh/Qi is the main subject of this report.

\section{Methods and material}

Since 2008 up to now several library studies has been conducted about the major concepts of Rouh/Qi in Traditional Medicine which lead to several publications. Some of them presented in national and international congress of traditional medicine and some other have been published in national and international journals. ${ }^{1-12}$

\section{Results and discussion}

\section{a. Electrical Skin Resistance}

Some parts of the skin have a different electrical skin resistance (ESR) from the surrounding region. ${ }^{13,14}$ These regions may be acupuncture points (APs).There are different known agents ${ }^{15,16}$ affecting ESR measurement out-come so that they may be variable in presentation.

\section{b. Acupuncture point}

What is the physiology of acupuncture point (AP) function? The Chinese style applies deep needling whereas the Japanese style apply superficial one, but therapeutic results are equal for both of them. ${ }^{17,18}$ The needling effect phenomena after acupuncture and by endorphin releasing, has been shown that acupunctures effect can last for many hours ${ }^{19}$ and its clinical positive effects can be maintained over a long period of time, ${ }^{20}$ even after the discontinuation of acupuncture sessions. ${ }^{21}$ There must be some physiological activities which occur at APs in contrast with their adjacent areas.

\section{c. Structure of primo vascular system (PVS) as base for rouh/ Qi production}

For more information about PVS, it is highly suggested to go to previous publications. ${ }^{1-12,22,23}$ A known scholar, called Bong Han Kim, in 1960 discovered a novel circulatory system which is a body wide web of vascular thread like structures in animal model having a floating fluid that called Bong-Han-fluid. The system is not visible by light microscopes or magnifying glasses but the system, only under phase contrast microscope, is detectable and thin only barely which are almost non-separable from fibrins in clot. ${ }^{22-25} \mathrm{Kwang}$-SupSoh et al. discovered that trypan blue is a useful dye for staining the structures of BHD. The name, PVS has been used instead of BHD since 2009 during a congress about the BHD. ${ }^{22-25}$ PVS include some ducts and corpuscles that together make a threadlike wide- web structure which each duct include sub ductules and this contain fluid with unidirectional movement. PVS system includes Primo Vessels (PV) or ducts and Primo Nodes (PN) or corpuscles that match with APs and AP Meridians (AM) respectively. PVS system is a flexible tissue, and once broken, it con curl and shrink. Sub networks of PVS include: (1) superficial, (2) intravascular, (3) intra vascular, (4) organ surface, (5) intra organ, (6) neural. ${ }^{1-4,16,25}$ According to this concept a network of this wide-web threadlike structure connect all the cells and points of the body to each other as "coherent body". ${ }^{1-4}$ PVS in details: PVS system differs from lymphatic and blood vessels. ${ }^{26}$ The histological of PVS structure appears simple and are organized from a bundle of several ductules, showing characteristic rod- shaped nuclei (10-20 $\mu \mathrm{m}$ in length). ${ }^{27}$ 
In cross- section, the PVS appeare as a small tissue formation, containing some small lumens $\left(6-10^{\mu m}\right)$ in diameter. The lumen of the ductule includes a single layer of endothelial cells enclosed by an extracellular matrix. ${ }^{1-4,27}$ A specific staining (DNA specific dye) was used to show inter granular fluid. PVS liquid contain: neurotransmitters, hyaluronic acids, free nucleotides, amino acids and adrenalin and noradrenalin. Additional significant role of liquid is the dispersion of electrical signs throughout the PVS system, which could provide a structure based issue for the well-known phenomenon of low electrical obstructions at AP. ${ }^{28}$ A hypothetical function of the liquid is light circulation which may explain the almost immediate impressions felt throughout the entire body when some needling is conducted at $\mathrm{AP}^{24}$

\section{d. Liquid flow direction}

A novel fluid flowing was discovered by injecting fluorescent nanoparticles into an organ surface PN. ${ }^{29}$ It has been shown that injecting alcian blue into a PN on the surface of a rabbit liver, can measure the average flow speed, ${ }^{30}$ and in agreement with bon hang Kim' s data it was $0 / 3 \pm 0 / 1 \mathrm{~m} / \mathrm{s} .{ }^{31}$ Movement of fluid in PVS: Contraction mechanisms of PN cells are induced by ca- ion channels and this was confirmed by testing with $\mathrm{Ca}$-ion-blocker (nifedipine) ${ }^{32}$ By the slow speed of this fluid, PVS can do regular checking and control all of body cells function.It may be suggested that increasing inter tissue pressure would affect PVS walls, leading to fluid leak, or even leading to fluid obstruction. This issue could open a field of discussion in acupuncture as Qi deficiency and or stagnation.

\section{e. Immune cells in PVS}

The PN and PV have an important number of Monocytes, Mastcells, Macrophages and Eosinophils. 23,27,33 Intra vascular BHD $(\mathrm{PV})$ is another hematopoietic system similar to bone marrow. ${ }^{34}$ There are many cells in the PVS like small and large lymphocytes, Monocytes, Granulocytes ${ }^{35,36}$ and all maturation stages of RBCs except normoblasts and mature RBCs.

\section{f. primo micro cells (PMCs)}

The pluri-potent ability to divide and differentiate into many kinds of cells in the same way that adult stem cells are able to do. ${ }^{37}$ The circulated pluri-potent stem cells in PVS could be transformed to adult stem cells with the same function for tissue regeneration. ${ }^{37}$

\section{g. budding method as a model for PMCs replication}

By protrusion of nuclei from PMCs and filament appearance and finally body process could lead to produce daughter cells. ${ }^{38}$

\section{h. cancer and PVS}

PVS has close relation with cancerous state. A recent Piece of research has shown that after two weeks of inoculation of human lung cancer cells in a mouse model subcutaneously, grown cancerous cells were found to be present and then, after a resection of the skin, the PVS were visible barely, in other side the PVS structures was fully revealed after a trypan blue staining. ${ }^{39}$ Recently there is suggested PVS as a rout for metastasis of cancerous cells. ${ }^{40}$ PVS which was presented as a theory for a new way for metastasis distribution, ${ }^{1-5}$ is approved by recent study. ${ }^{40}$ This is an idea which claims PVS control apoptosis and sporosis, so that when this process is out of control can lead to growth of cancerous mass. Also this tumoral mass is wrapped by PVS, but in contrast to other cells, there is no any control on tumor and in other way PVS can be used for metastasis of tumoral cells. ${ }^{1-5}$

\section{Mechanisms of rouh/Qi production in PVS}

Is energy stimulation by acupuncture true or false? A photon is an elementary particle which may be the quantum of light or other forms of electromagnetic radiation and therefore is as a carrier of electromagnetic force. ${ }^{41}$ Photon has wave-particle duality which exhibits properties of both energy and material. ${ }^{41}$ This meaning of photon can be observed and presented in human body as bio photon or Qi or Dameh. ${ }^{3-5}$ By emission of biophotons they are visible and can be detected in various part of human body. ${ }^{42-48}$ In our previous reports, these kinds of bio photon emission can be attributed to the Qi production and emission which is related and connected at AP effects. ${ }^{1-5}$

\section{a. DNA (flowing in PVS) as a source of bio photons}

An exited atom or molecule emits light when an electron, having captivated (absorbed) a quantum of energy, is raised to a high level of energy. A DNA molecule is an exciplex in which photons are stored and can be a source of bio photons. Exciplex formation in DNA has been show in various conditions, even at room temperature. ${ }^{24,36}$ Non coding DNA may act as a photon store and a coherent radiator, because of its enormous polymer size and its ability to form exciplexes, the biophysical model for intra and extra cellular communication developed by Nagl and Popp. ${ }^{49}$ Postulated that the bio photons is trapped and emitted by a cellular physical resonance device, namely DNA flowing in PVS, which results in bio photon emissions with a higher degree of coherence. The PVS presented in our previous report by ${ }^{1,5}$ having DNA granules running inside PVS and as the base of channels of AP, which are spread all over the body, can be like as a network for distribution and spreading of DNA molecules (as an exciplex) and non-coding DNA (as a photon store), so that by this network AP effects of bio photon or Qi production can be easily distributed and observed in long distances. ${ }^{1-5}$ In other word, DNA included in the PMC could be the source and storing place of bio photons which could be suggested to be called Rouh/Qi. Based on this concept, any Rouh/Qi (Bio photons) producing stimulators for example light (laser or Infra-Red radiation), movement (massage), heat (moxabustion), needling, and so on could stimulate the production of Rouh/Qi. ${ }^{1-5}$

\section{Produced rouh/Qi}

The oxidative metabolism processes of cells produces reactive oxygen species (ROS) that lead to the excitation of constituents of cells that gives rise to them emitting ultraweak light. ${ }^{50}$ The biophoton emission resulted from a lack of activity on the part of the antioxidant agent in protecting the cells from ROS. ${ }^{51}$ ATP releasing metabolism of mitochondria has an important role in producing ROS. ${ }^{52}$ The intracellular proteins are the source of the energy (ATP) transforming to Ultraweak Photon Emission (UPE). There are several agents that induce UPE. ${ }^{53}$ Oxidation induced UPE is dominated by proteins and along with oxidized amino acids is the main source of emitted photons. ${ }^{54}$ UPE may be different under various conditions such as the temperature of the skin, $\mathrm{O} 2$ concentration, humidity and $\mathrm{PH} .{ }^{55}$ This UPE may be as smallest part of Rouh/Qi. ${ }^{1-5}$ Noncoding DNA (circulating in the PVS fluid), as a photon store can be responsible for Rouh/Qi movement. ${ }^{1-5}$ 
If the PVS system were the basic concept of Rouh/Qi production (and movement) in acupuncture meridians $\mathrm{s}^{2-3,56,57}$ then acupuncture needling or laser acupuncture would induce photon (Rouh/Qi) emission from Acupuncture points (Aps). ${ }^{58,59}$ Rouh/Qi after production will move along with subductule of PVS. This is called Rouh/Qi movement which via the PVS and reaches to the cellular level and keeps cells, tissues, organs and human body alive. The movement of Rouh/Qi, in Traditional Medicines (PM and TCM), leads to viability and aliveness in human body. The most APs are found at connective tissue planes. ${ }^{57} \mathrm{~A}$ variety of cells and tissue, as an extracellular matrix, form the connective tissue.

\section{Rouh/Qi after production}

Rouh/Qi movement could be normal in PVS or may be affected by some factors and be presented as an excess or deficiency. ${ }^{3-5}$ Conductivity in connective tissue is sensitive to mechanical pressures, $\mathrm{PH}$ and ionic composition. ${ }^{60,61}$ It increases significantly in the case of increased water absorption. ${ }^{3-5,61}$ Weak signals of mechanical pressure, heat or electricity may be readily amplified and propagated. ${ }^{3-5,61}$ The PVS network as a base provides normal qi movement, however, some mechanisms such as "oriented nature of collagen liquid crystalline" have been proposed and can amplify and propagate the weak signals. ${ }^{62}$

\section{Objective appearance of rouh/Qi}

As a specific diurnal pattern of UPE / Rouh/Qi emission intensity is low during the day, rises in the evening and is high at night for both dorsal and palmar sides. There is the shifting manner between the left and right sides. ${ }^{63,64}$ As a pattern we can describe that the intensity increases on the left (Yin) hand starting around $8 \mathrm{PM}$ and from 12 $\mathrm{PM}$ until $5 \mathrm{AM}$ the left hand emits more than the right. The right side (Yang) has a pattern as a complementary role in comparison to the left side (Yin). ${ }^{63,64} \mathrm{~A}$ diurnal pattern is related with the season, month, time, gender, individual and circadian cycle. ${ }^{65}$ This pattern is specific in individuals and is different in every person (individual Rouh/Qi). The temperature or blood circulation does not cause changes in this pattern of personal photon emissions. Somehow, the pattern of cortisol concentration in human body has a positive correlation with Yin pattern. According to this pattern Cortisol concentration is a kind of Yin cycle and acts like it and has the fluctuation pattern. ${ }^{65}$ The diurnal pattern has demonstrated by using a sensitive charge coupled device (CCD) camera ${ }^{65,66}$ Of course earlier study has an idea in revers to this idea. Moxibustion or infrared light stimulation may show different structures appearance on thermographic images of the human body. They may be technical artifacts and are not identical to what are known as meridians. But it suggests that further scientific studies are required regarding the possible visualization of meridians. ${ }^{66}$

\section{Similarity of rouh/Qi and dameh (bokhar): common area of TCM and PM}

Qi production was described in detail. There is a similarity between Qi and Rouh in TCM and PM. Rouh and Qi have similar meaning in both TCM and PM. Based on PM concept, Rouh (other name in PM are Dameh or Bokhar) is a kind of gaseous steam, separated from any liquid or solid substance, can be produced by for example heat and has an ascending movement to a higher level. Qi movement in TCM is similar to Rouh (Dameh/Bokhar) movement in PM. This similarity suggests that photon/Qi and Rouh have unique and similar properties.
In other words can be shown that Dameh/Bokhar is smallest part of Rouh (like a cloud) and Rouh (cloud) is a collection of Dameh. ${ }^{4}$ This is true in TCM which qi is smallest particle of Qi and Qi is the collection of qi. ${ }^{4}$

Photon/qi/Dameh/Bokhar (smallest particle of Qi/Rouh) has an invisible movement per se (at the atomic level) which is named Gohari's (innate) movement in $\mathrm{PM}^{4}$ or original movement in TCM. This invisible movement may have non-directional movement and in the form of particle there is not any visible effect whereas in the form of a collection of particles (Rouh/Qi) may have a directed or planned movement which can be called Qi movement in TCM or Rouh in PM (Rouh/Qi). ${ }^{4}$ In other words, Rouh/Qi is the movement of a collection of qi/photon/Dameh.

In other word; the meaning of "free flow of qi" in TCM can be similar to meaning of Movement of Gohari (Innate) in PM, but the directed movement of gathered qi can be called Rouh/Qi movement. ${ }^{4}$ As be reported, the life state is a fluctuation of Yin-Yang pattern. ${ }^{66}$ We claim that fluctuation of Rouh/Qi is which lead to fluctuation of Yin-Yang pattern of life state and is considered as a pixel of life which form life puzzle. In fact the picture of life is made of small pieces of pixels which are in fluctuation and lead to fluctuation of mental and physical state of human body. ${ }^{4}$

In fact, the body is made of milliards of milliard of qi with having "free flows of qi" (similar to a pixel of a picture) which by gathering altogether a collection of Qi (Dameh) which can produce the picture of the body as Rouh/Qi. Rouh/Qi is an individual subject. Individualization of Rouh/Qi may depends on underlying factors of any person which is different from person to person so that provide various persons with different features which is PM/TCM is called as Mezaj/Zheng. ${ }^{4}$ For example, in Phlegmatic Mezaj/Zheng persons (lazy and slow motion person), anger (rapid movement of Rouh/ Qi) may help the physiologic manner of individual for qi movement nevertheless anger, could be pathologic in Choleric Mezaj/Zheng persons (hot-blooded and fast movement) and leads to hypertension. ${ }^{4}$ The differences depend on Mezaj/Zheng which is underlying issue for precision medicine. ${ }^{4}$

\section{What may be mezaj/zheng?}

According to the concepts of TCM, Yin, Blood, Yang and qi can be compared to Cold, Wetness, Heat, and Dryness in PM. Solid is dry and fluid is wet. Heat (Yang) produces dryness (qi). In other words dryness in PM can be similar to the qi concept of TCM. Yin (protein or collagen, cold), Blood (plasma or serum, wetness, moistness), Yang (heat or fire, hot) are the underlying factors for qi production. Heat induces production of qi. ${ }^{62}$ and water facilitates the movement of qi. ${ }^{55}$ Yin (protein) is considering as a source. ${ }^{3-5}$ So this collection makes an integrated holistic body which is called Mezaj in PM and Zheng in TCM. Every person has Mezaj-based behaviors, speech, thinking, mind, and even physical appearance. The quality and quantity of the Mezaj/Zheng is different in every individual in different situations. ${ }^{4}$

\section{Original Qi (dameh,gohar) as a base of life}

Gohar or original Qi are fundamental concepts in TCM and PM. They are para-substance, para-existence and or subjective properties. In other words they are metaphysical. Surely this concept is a new science and there are a lot of question to be answered, such as does 
the reinforcing or reduction of acupuncture points have a placebo effect or a real effect? Is there any relationship between the channels of acupuncture or between the upper and lower parts of the body? There are many other critical questions which must be answered if we are to understand the mechanisms of acupuncture such as which organ or organs of the five elements are the source of the life cycle? What and where is San Jiao? The subject which is presented in our previous report of Idea ${ }^{5,6}$ and recently there are two more publications to review on the San Jiao. ${ }^{11,12}$ What is the map of PVS? These and thousands of questions must be answered by futures research. For the first time we suggested that PVS can be a rout for metastasis spreading, ${ }^{4,11,12}$ which has been recently approved. ${ }^{40}$ We believe that PVS has a map which is critical for life style, health and diseases state of human body. If we were to have a map of PVS could be able to control Rouh/Qi and this could be directed to target organs, which is the main purpose of acupuncture.

\section{Conclusion}

Life and living is complicated process which may have different definitions in Traditional Medicine and also western medicine. Rouh/ Qi is the basic material of life which has been considered lesser than other issue of life. This review reports how Rouh/Qi (energy) is produced and how find an actual meaning, then it transported by special way (PVS) and by reaching to all cells of human body, it will transformed into the energy for function.

\section{Acknowledgements}

Special thank all the journals which helped to publish my theory (1-12) since 2008 up to now.

\section{Conflict of interest}

There is not any conflict of area.

\section{References}

1. Avijgan M. A brief review of the BHD as base for acupuncture function. Book abstract of $7^{\text {th }}$ International Congress of Medical-Cosmetic Acupuncture and 312 meridian exercises. Aurangabad: India; 2012. p. $142-148$.

2. Avijgan M. Isfahan University of Medical Sciences. Iran | Traditional Medicine-2014 | OMICS group;2014.

3. Avijgan M, Avijgan M. Can the Primo Vascular System (Bong Han Duct System) be a Basic Concept for Qi Production? Int J Integr Med. 2012;(20).

4. Avijgan M, Avijgan M. The Infrastructure of the Integrative Human Body; Qi/Dameh, Qi Movement/ Rouh and Zheng/ Mezadj-Scientific Base. Int J Integr Med. 2013;1(22):2013.

5. Avijgan M, Avijgan M. Fasciology as Base of Traditional Medicine. National congress of Traditional Medicine at Isfahan University of Medical Sciences. Iran: 2017.

6. Avijgan M. Triple burner or San jiao as a real or false space of body. Alternative \& Integrative Medicine. 2014.

7. Avijgan M, Avijgan M. Does the Primo Vascular System Originate from the Polar Body. Integr Med Int. 2014;1:108-118.

8. Avijgan M, Avijgan M. Meraque or Triple Energizer (San Jiao): Actual or Virtual Organ in Traditional Medicine - A Hypothetical Viewpoint. Integr Med Int. 2015;2:9-20.

9. Avijgan M. Salehzadeh F, Kamran AA, et al. Concept of Infectious Diseases in Persian Medicne. Advances in Infectious Diseases. 2017;7:1-10.

10. Avijgan M, Mazaheri M, Salehzadeh F. Comparison between clinical findings of Infectious Diseases in Traditional Persian Medicine (Tibbe Akbari) and Modern Medicine (Harrison textbook). Journal of Tibbe Sonati of Islam and Iran. 2017;8(2):155-160.

11. Avijgan M, Avijgan M. Mesentery System is a Section of Meraque System in Persian Medicine. Int J Complement Alt Med 2017;8(5):00275.

12. Avijgan Majid, Avijgan Mahtab, Salehzadeh F. Meraque/San Jiao of Eastern Medicine in Comparison with the Mesentery System Presented in Western Medicine. Integr Med Int. 2017;4:101-106.

13. Nakatani Y. Skin electric resistance and Ryodoraku. J Autonomic Nerve. 1956;6:52-83.

14. Niboyet J. Nouvelle constatations sur les proprietés electriques des ponts Chinois. Bull Soc Acup. 1985;30:7-13.

15. Lawler JC, Davis MJ, Griffith EC. Electrical characteristics of the skin. The impedance of the surface sheath and deep tissues. $J$ Invest Dermatol. 1960;34:301-308.

16. Ahn AC, Martinsen OG. Electrical characterization of acupuncture points: technical issues and challenges. J Altern Complement Med. 2004;13(8):817-824.

17. Hui KK, Liu J, Makris N, et al. Acupuncture modulates the limbic system and subcortical gray structures of the human brain: evidence from fMRI studies in normal subjects. Hum Brain Mapp. 2000;9(1):1325 .

18. Hui KK, Liu J, Marina O, et al. The integrated response of the human cerebrocerebellar and limbic systems to acupuncture stimulation at ST36 as evidenced by fMRI. Neuroimage. 2005;27(3):479-496.

19. Han JS. Acupuncture and endorphins. Neurosci Lett. 2004;361(13):258-261.

20. Thomas KJ, MacPherson H, Thorpe L, et al. Randomised controlled trial of a short course of traditional acupuncture compared with usual care for persistent non-specific low back pain. BMJ. 2006;333(7569):623-626.

21. Mac Phersona H, Greenb G, Nevadob A, et al. Brain imaging of acupuncture: Comparing superficial with deep needling. Neuroscience Letters. 2008;434(1):144-149.

22. Lee BC, Kim KW, Soh KS. Visualizing the network of Bonghan ducts in the omentum and peritoneum by using Trypan blue. $J$ Acupunct Meridian Stud. 2009;2:66-70.

23. Lee BC, Yoo JS, Ogay V, et al. Electron microscopic study of novel threadlike structures on the surfaces of mammalian organs. Microsc Res Tech. 2007;70(1):34-43.

24. Soh KS. Bonghan Duct and Acupuncture Meridian as Optical Channel of Biophoton. Journal of the Korean Physical Society. 2004;45(5):1196-1198.

25. Kwang-Sup Soh. Bonghan Circulatory System as an Extension of Acpuncture Meridians. Journal of Acupuncture and Meridian Studies. 2009;2(2):93-106.

26. Lee BC, Baik KY, Johng HM, et al. Acridine orange staining method to reveal the characteristic features of an intravascular threadlike structure. Anat Rec B New Anat. 2004;278(1):27-30. 
27. Ogay $\mathrm{V}$, Bae $\mathrm{KH}$, Kim KW, et al. Comparison of the characteristic features of Bonghan ducts, blood and lymphatic capillaries. J Acupunct Meridian Stud. 2009;2(2):107-117.

28. Ahn A, ColberAP,Anderson BJ, et al. Electrical properties of acupuncture points and meridians: a systematic review. Bioelectromagnetics. 2008;29(4):245-256.

29. Lee $\mathrm{CH}$, Yoo JS, Kim $\mathrm{HH}$, et al. Flow of nanoparticles inside organs-surface Bonghan ducts. Proc 23rd Sym Kor Soc Jungshin Sci. 2005;23:129-134.

30. Sung B, Kim MS, Lee BC, et al. Measurement of flow speed in the channels of novel threadlike structures on the surfaces of mammalian organs. Naturwissenschaften. 2008;5:117-124.

31. Kim BH. The Kyungrak system. J Jo Sun Med. 1965;108:1-38.

32. Park SH. Bioelectrical Study of Bonghan System. Ph.D. Thesis, South Korea:Seoul National University; 2009.

33. Yoo JS, Kim MS, Sung B, et al. Cribriform structure with channels in the acupuncture meridianlike system on the organ surfaces of rabbits. Acup Electrother Res. 2007;32:130-2.

34. Kim BH. Sanal and hematopoiesis. J Jo Sun Med. 108:1965;1-6.

35. Shin HS, Johng $\mathrm{H}$, Lee BC, et al. Feulgen reaction study of nove threadlike structures on the surface of rabbit livers. Anat Rec B New Anatomist. 2005;284(1):35-40.

36. Dini L, Coppola S, Ruzittu MT, et al. Multiple pathways for apoptotic nuclear fragmentation. Exp Cell Res. 1996;223(2):340-347.

37. Becker AJ, McCullough EA, Till JE. Cytological demonstration of the clonal nature of spleen colonies derived from transplanted mouse marrow cells. Nature. 1963;197:452-454

38. Kim BH (1965) The Sanal theory. J Acad Med Sci DPR Korea. 1965;108:39-62.

39. Yoo JS, Kim HB, Ogay V, et al. Bonghan ducts as possible pathways for cancer metastasis. J Acupunct Meridian Stud. 2009;2:118-123.

40. https://www.researchgate.net/publication/44692372

41. http://en.wikipedia.org/wiki/Photon

42. Kim JD, Choi C, Lim JK, et al. Biophoton emission from rat liver. $J$ Korean Phys Soc. 2003;42:427-430.

43. Barsaschi R. Biochem, Biophysics. Acta. 1983;762:241.

44. Cadenas E, Arad I.D, Boveris A, et al. Partial spectral analysis of the hydroperoxide-induced chemiluminescence of the perfused lung. FEBS Lett. 1980;111(2):413-418.

45. Artem'ey VV, Goldobin AS, Gus'kov LN. Recording the optical emission of a nerve. Biophysics. 1967;12:1278-1280.

46. Blokha VV, Kossova GV, Sizov AD, et al. The ultraweak glow of muscles on stimulation. Biophysics. 2014;13:1084-1085.

47. Cohen S, Popp FA. Biophoton emission of the human body. J Photochem Photobiol B Biol. 2003;41(5):187-189.

48. Kim TJ, Shin HS, Lee SM, et al. Biophoton Emission from Fingernails and Fingerprints of Living Human Subjects. Acupunct Electrother Res. 2002;27(1-2):85-94.

49. Langevin HM, Yandow JA. Relationship of acupuncture points and meridians to connective tissue planes. Anat Rec $B$ New. 2002;269(6):257-265.
50. Kobayashi M, Takeda M, Ito K, et al. Two-dimensional photon counting imaging and spatiotemporal characterization of ultraweak photon emission from a rat's brain in vivo. Journal of Neuroscience Methods. 1999;93(2):16-168.

51. Boveris A, Cadenas E, Reiter R, et al. Organ chemiluminescence: Noninvasive assay for oxidative radical reactions. Proc Natl Acad Sci USA. 1980;77(1):347-351.

52. Cadenas E, Boveris A, Chance B. Low-level chemiluminescence of biological systems. In: Proyor WA, editor. Free Radicals in Biology, vol. 6. New York: Academic Press; 1990. p. 211-242.

53. Hagens F, Khabiri F, Schreiner V, et al. Non-invasive monitoring of oxidative skin stress by ultraweak photon emission measurement. II: biological validation on ultraviolet A-stressed skin. Skin Res Technol. 2008;14(1):112-120.

54. Khabiri F, Hagens R, Smuda C, et al. Noninvasive Monitoring of Oxidative Skin Stress by Ultraweak Photon Emission (UPE)Measurement. I: mechanisms of UPE of biological materials. Skin Res Technol. 2008;14(1):103-111.

55. Ou-Yang H, Stamatas G, Saliou C, et al. A cheminluminescence study of UVA-induced oxidative stress in human skin in vivo. J Invest Dermatol. 2004;122(4):1020-1029.

56. Ho MW, Knight DP. The acupuncture system and the liquid crystalline collagen fibers of the connective tissues. Am J Chin Med. 26 1998;26(3-4)251-263.

57. Langevin HM, Yandow JA. Relationship of acupuncture points and meridians to connective tissue planes. Anat Rec. 269(6): 257-265.

58. Inaba H. Measurement of ultra-weak biophotons information. Proc Inst Electrostat Japan. 1998;22:215-252.

59. Inaba H. Human sensing based on ultimate Technology of optical measurement .Opt Electroopt Eng Contact. 1999;37:251-267.

60. Van Wijk R, Souren JEM, Ovelgonne H. The extracellular matrix, fibroblast activity and effect of Echinacea purpurea. In: H Heine, G Anastasiadis, editors. Normal Matrix and Pathological Conditions. Fisher Verlag, Stuttgart: German.

61. Leikin S, Rau D C, Parsegian VA. Temperature-favoured assembly of collagen is driven by hydrophilic not hydrophobic interactions. Nat Struct Biol. 1995;2(3):205-210.

62. Mikhailov AS, Ertl G. Nonequilibrium Structures in Condensed Systems. Science. 1996;272(5268):1596-1597.

63. Cifra M, Van Wijk EPA, Koch H, et al. Spontaneous ultraweak photon emission from human hands is time dependent. Radioengineering. 2007;16:15-19.

64. Van Wijk E, Van Wijk R, Cifra M. Spontaneous ultra-weak photon emission from human hands varies diurnally. In: Popp J, Von Bally G, editors. Proc Spie. 2012;6633:66331J1-66331J9.

65. Van Wijk R, Van der Greef J, Van Wijk E. Human Ultraweak Photon Emission and the Yin Yang Concept of Chinese Medicine. J Acupunct Meridian Stud. 2010;3(4):221-231.

66. Kobayashi M, Kikuchi D, Okamura H. Imaging of Ultraweak Spontaneous Photon Emission from Human Body Displaying Diurnal Rhythm. PLoS One. 2009;4(7):e6256.

67. Litscher G. Infrared thermography fails to visualize stimulationinduced meridian-like structures. Biomed Eng Online. 2005;4:38.

68. Laakso M, Björk BC. Anatomy of open access publishing: a study of longitudinal development and internal structure. BMC Med. $2012 ; 10: 124$ 